\title{
Spectroscopy of the Cadmium isotopes
}

\author{
John L. Wood \\ School of Physics, Georgia Institute of Technology, Atlanta, GA 30332-0430, USA
}

Abstract. The cadmium isotopes have now been characterized across the entire $50<\mathrm{N}<82$ shell. A brief review is given of the identifiable structures. Some discussion of open questions is made, especially of vibrations.

\section{Introduction}

The cadmium $(Z=48)$ isotopes have been a focus of interest in nuclear structure for many decades. This is because they exhibit collective excitations and are located adjacent to the closed shell at $\mathrm{Z}=50$.

At the closed neutron shells, $\mathrm{N}=50,82$, pairing dominates and is manifested as excitations with good seniority. Moving towards the mid-neutron shell at $\mathrm{N}=66$, collectivity emerges; but exactly how and what kind is an open question. In the vicinity of the mid-shell, the former view of the structure of the $\mathrm{Cd}$ isotopes was one of near-harmonic quadrupole collective vibrations: this view has been refuted [1]. However, exactly what is the collective character of the mid-shell $\mathrm{Cd}$ isotopes, remains an open question. The present paper discusses these issues.

\section{A global view of the $\mathrm{Cd}$ isotopes}

Figure 1 presents seniority structures due to the $\pi g_{9 / 2}{ }^{-2}$ configuration as manifested in ${ }^{98-130} \mathrm{Cd}$. Figure 2 presents

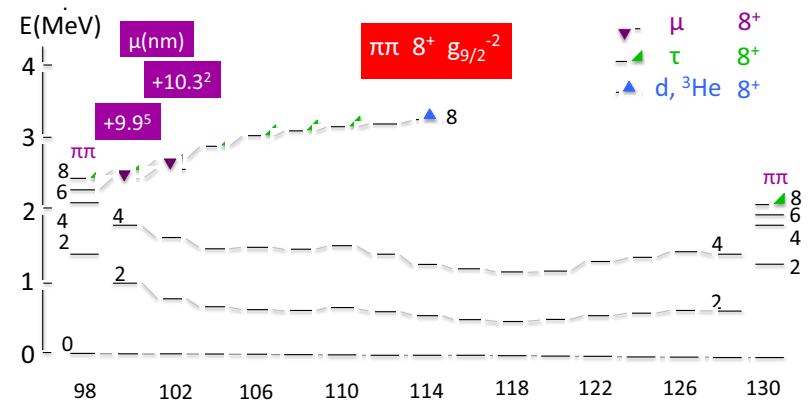

Figure 1. Seniority structures in the $\mathrm{Cd}$ isotopes resulting from $\pi \mathrm{g}_{9 / 2}{ }^{-2}$ configurations. The data are taken from Nuclear Data Sheets.

seniority structures due to the $\mathrm{vg}_{7 / 2}{ }^{+2}, \mathrm{vg}_{7 / 2} \mathrm{vd}_{5 / 2}$, and $v \mathrm{~h}_{11 / 2}{ }^{-2}$ configurations as manifested in ${ }^{100-128} \mathrm{Cd}$. These are characterized spectroscopically by one-nucleon transfer reactions, magnetic moments, and lifetimes.

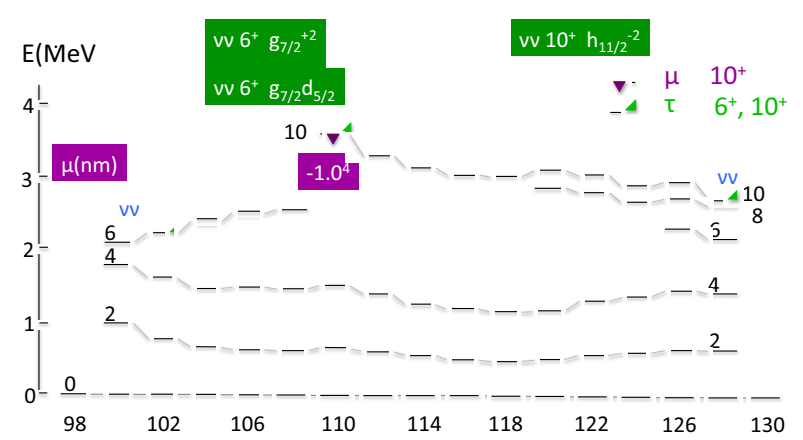

Figure 2. Seniority structures in ${ }^{102-128} \mathrm{Cd}$ resulting from $v \mathrm{~g}_{7 / 2}{ }^{+2}$, $v h_{11 / 2}{ }^{-2}$, and $v g_{7 / 2} v d_{5 / 2}$ configurations. The data are taken from Nuclear Data Sheets.

In the mid-shell region of the $\mathrm{Cd}$ isotopes shape coexistence is well-established and is interpreted as resulting from a proton-pair excitation across the $Z=50$ shell gap [2]. This is shown in Fig. 3.

The major open question is the nature of the collective structures built on the Cd ground states. Maps

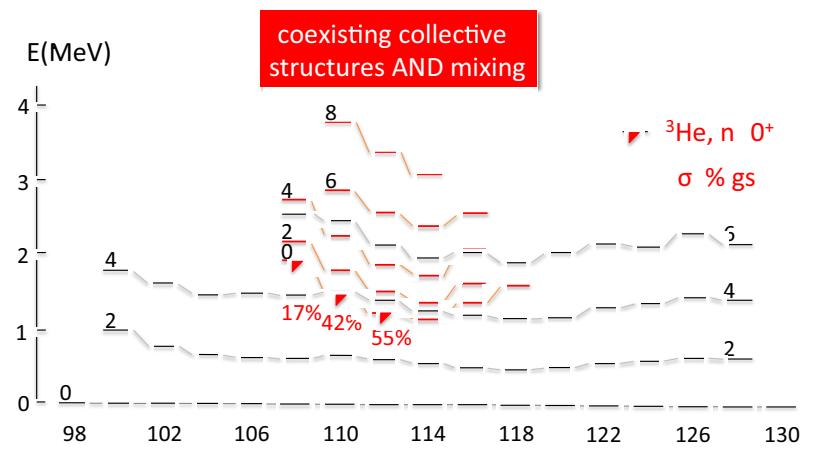

Figure 3. Deformed structures in ${ }^{108-118} \mathrm{Cd}$ resulting from a $\pi(2 \mathrm{p}-$ 4h) configuration. The data are taken from Nuclear Data Sheets.

of $B(E 2)$ values provide a powerful summary of the collective character of a series of isotopes. These are

\footnotetext{
${ }^{\mathrm{a}}$ Corresponding author: john.wood@physics.gatech.edu
} 
shown for the Cd isotopes in Figs. 4, 5, 6, 7, 8, and 9. While the energy pattern of excited states suggests

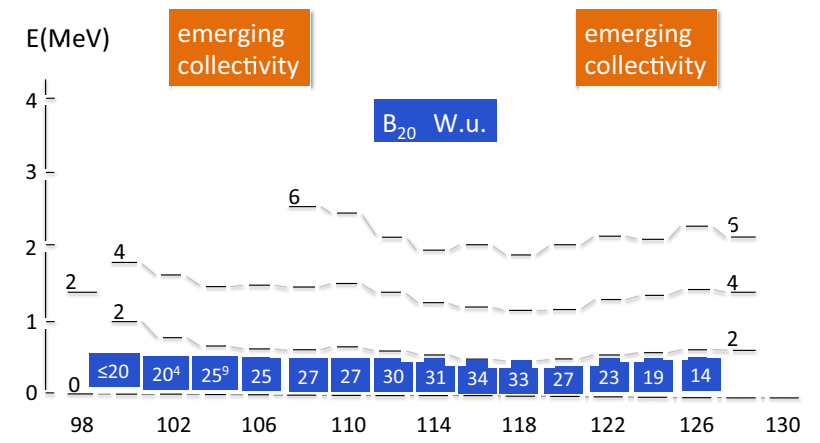

Figure 4. Map of $\mathrm{B}\left(\mathrm{E} 2 ; 2_{1}{ }^{+} \rightarrow 0_{1}{ }^{+}\right)=\mathrm{B}_{20}$ expressed in W.u. Collectivity is evidently emerging by ${ }^{102} \mathrm{Cd}$ and ${ }^{124} \mathrm{Cd}$. The data are taken from Nuclear Data Sheets and [3,4].

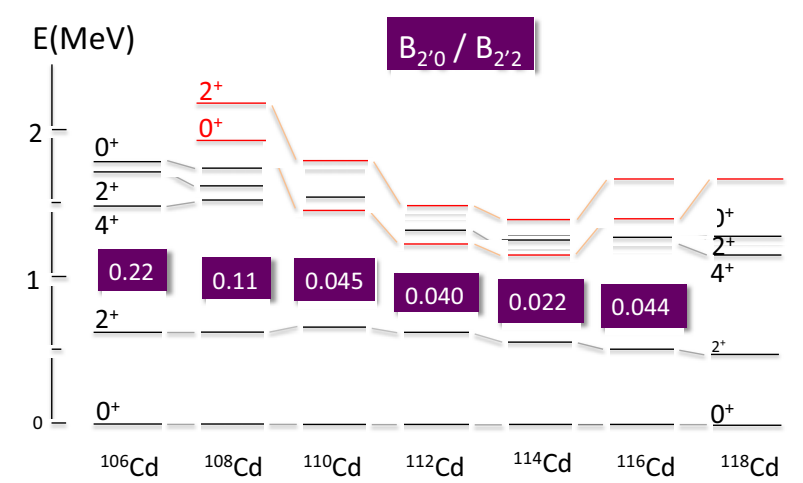

Figure 5. Map of $\mathrm{B}\left(\mathrm{E} 2 ; 22^{+} \rightarrow 0_{1}{ }^{+}\right) / \mathrm{B}\left(\mathrm{E} 2 ; 22^{+} \rightarrow 21^{+}\right)=$

$\mathrm{B}_{2}{ }^{\prime} / \mathrm{B}_{2}{ }^{\prime}$. Intruder states are shown in red. The data are taken from Nuclear Data Sheets.

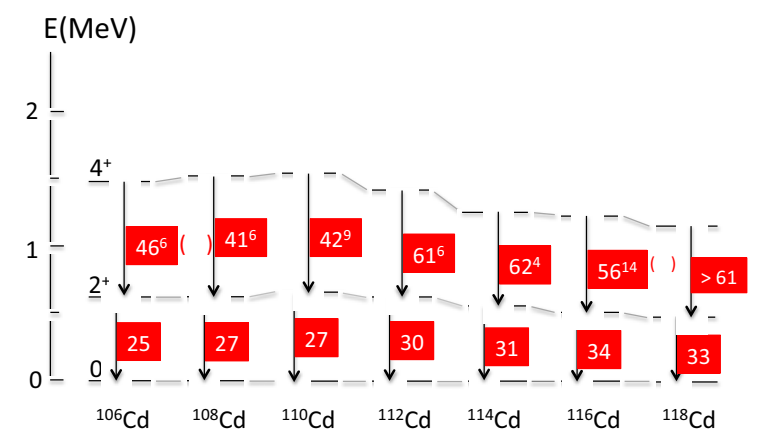

Figure 6. Map of B(E2; $\left.4_{1}{ }^{+} \rightarrow 2_{1}{ }^{+}\right)$and B(E2; $21^{+} \rightarrow 0_{1}{ }^{+}$) expressed in W.u. Good candidates for harmonic quadrupole vibrators are indicated by check marks. The data are taken from Nuclear Data Sheets.

near-harmonic quadrupole vibrational behavior, the $\mathrm{B}(\mathrm{E} 2)$ pattern does not support this. Undertaking a characterization of just what is the nature of the collective nuclear structure built on the $\mathrm{Cd}$ ground states has proven to be one of the most highly demanding tasks in nuclear spectroscopy, probably, that has ever been undertaken. Some details are given in the next section.

A further issue that arises in the $\mathrm{Cd}$ isotopes is the possibility of elucidating the emergence of collectivity from its incipient appearance. To this end, the spectroscopy of ${ }^{100-108} \mathrm{Cd}$ is of particular interest. This is the subject of the contribution by Andrey Blazhev to these Proceedings.

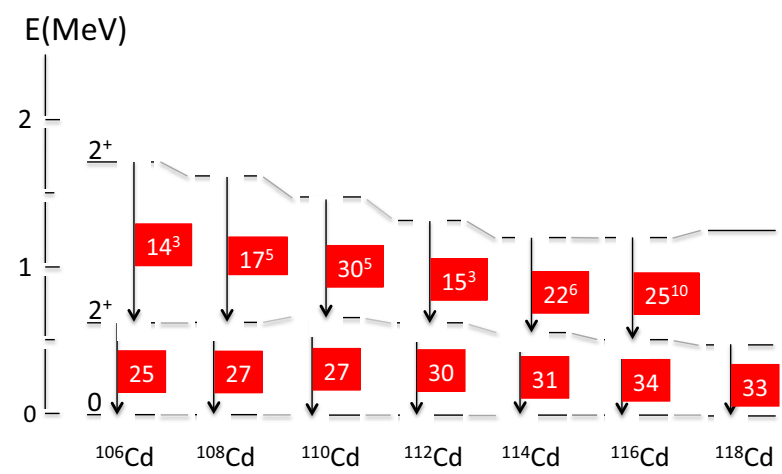

Figure 7. Map of $\mathrm{B}\left(\mathrm{E} 2 ; 22^{+} \rightarrow 21^{+}\right)$and $\mathrm{B}\left(\mathrm{E} 2 ; 21^{+} \rightarrow 0_{1^{+}}\right)$ expressed in W.u. The data are taken from Nuclear Data Sheets

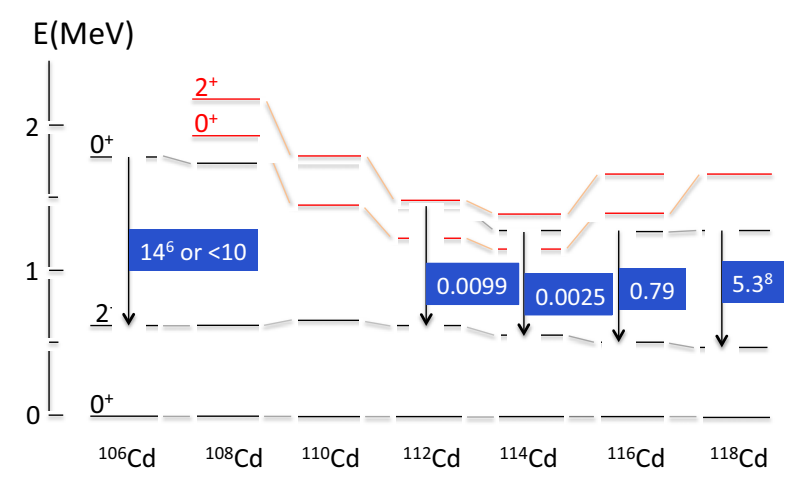

Figure 8. Map of $\mathrm{B}\left(\mathrm{E} 2 ; \mathrm{vvib}^{+} \rightarrow 21^{+}\right)$expressed in W.u. for $0^{+}$ states that are candidate two-phonon states. The data are taken from Nuclear Data Sheets.

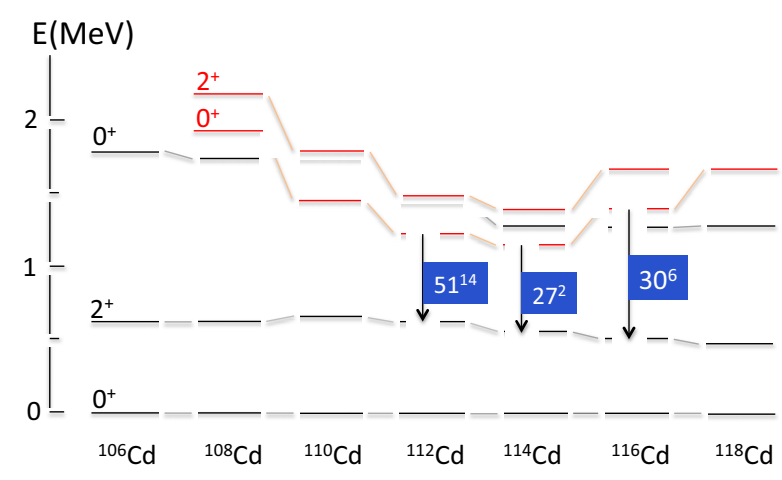

Figure 9. Map of B(E2; $\left.0_{\mathrm{def}^{+}} \rightarrow 21^{+}\right)$expressed in W.u. for $0^{+}$ states that are deformed states. The data are taken from Nuclear Data Sheets

\section{Detailed spectroscopic studies of the $\mathrm{Cd}$ isotopes}

Detailed spectroscopic data for the $\mathrm{Cd}$ isotopes have begun to be acquired, especially by the technique of inelastic neutron scattering as carried out at the Univ. of Kentucky Accelerator Laboratory [5-8] (and see the paper by Steven Yates in these Proceedings). These data have 
been combined with ultra-weak $\gamma$-ray decay branch measurements following $\beta$ decay $[9,10]$. An outcome of these studies is presented in Fig. 10, which summarizes pertinent data that are a first look beyond a vibrational interpretation of ${ }^{110-116} \mathrm{Cd}$.

To move beyond the present status of the structure of the $\mathrm{Cd}$ isotopes will be even more demanding of spectroscopic techniques. An obvious direction is multistep Coulomb excitation. At present, such data only exist for ${ }^{114} \mathrm{Cd}$ [11]. A less obvious direction is transfer reaction

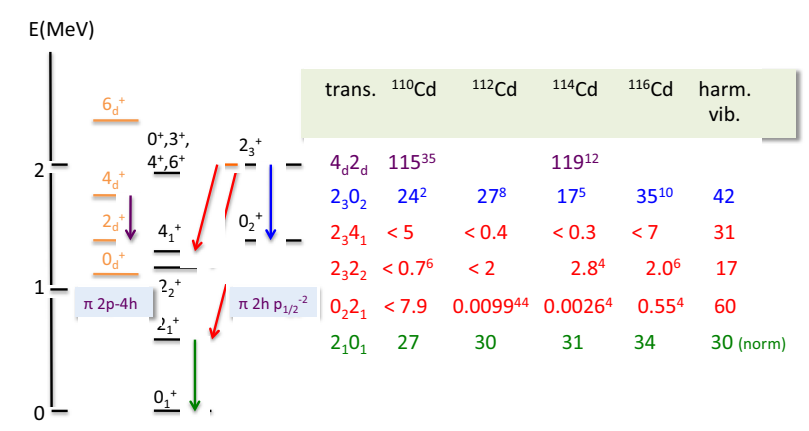

Figure 10. Electric quadrupole transition strengths in ${ }^{110-116} \mathrm{Cd}$ deduced from lifetime measurements and $\gamma$-ray transition intensities. The data are taken from Nuclear Data sheets and references given in the text.

spectroscopy, using both one- and multi-nucleon transfer (see the paper by Paul Garrett in these Proceedings). A particular outcome of such measurements is that they reveal the non-collective states in weakly collective nuclei such as the $\mathrm{Cd}$ isotopes.

A rarely conducted type of spectroscopy that has been carried out for many decades is conversion electron spectroscopy, which, besides the familiar outcome of transition multipolarities, is uniquely able to quantify E0 transition strengths (given that the lifetime of the parent level is known). Such strengths are a sensitive and modelindependent view of shape coexistence and mixing (see, e.g., [12]).

An analysis for the E0 transition strengths is presented here for ${ }^{114} \mathrm{Cd}$. It relies on having information for mixing amplitudes for $0^{+}$states from two-neutron transfer data [13]. The essential theory and the input data are summarized in Fig. 11. The extension of the mixing

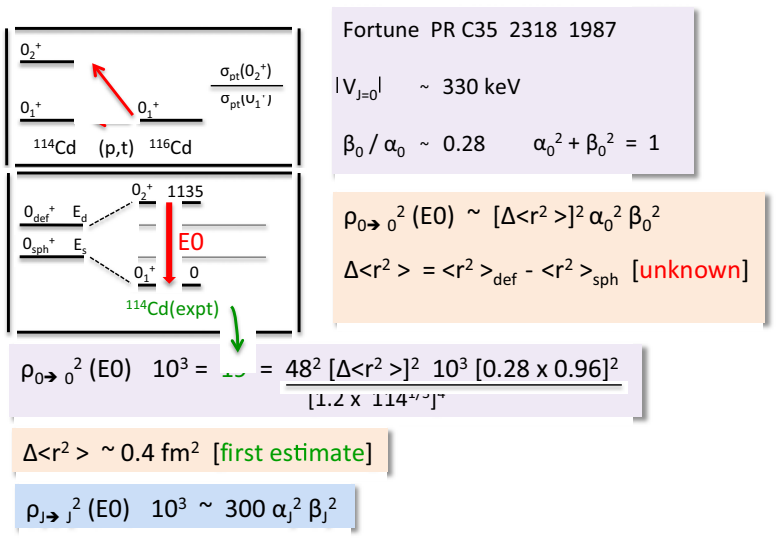

Figure 11. Electric monopole transition strength, $\rho^{2}(\mathrm{E} 0)^{*} 10^{3}$ in ${ }^{114} \mathrm{Cd}$ and its origin through configuration mixing.

analysis to states with spin other than zero is presented in Fig. 12. In particular, note that the input quantity, $\Delta<r^{2}>$

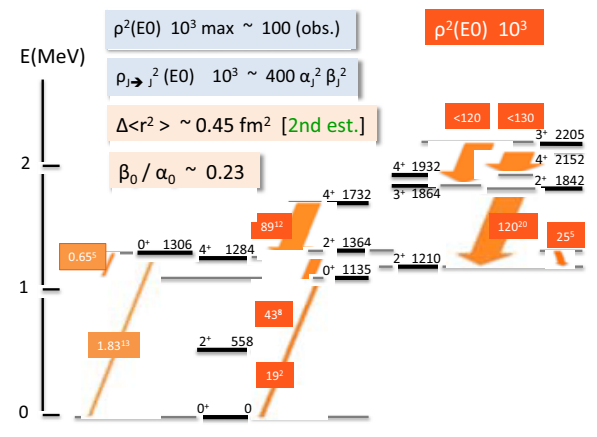

${ }^{114} \mathrm{Cd}$

Figure 12. Electric monopole transition strengths, $\rho^{2}(\mathrm{E} 0)^{*} 10^{3}$ in ${ }^{114} \mathrm{Cd}$ for all strong $\mathrm{E} 0$ transitions and the fine-tuning of its strength (see text). The data are taken from $[12,14]$.

is taken to be spin independent and is fine-tuned to the largest observed value of $\rho^{2}(\mathrm{E} 0)^{*} 10^{3}$ and the presumption that this corresponds to $\alpha=\beta=0.50$, i.e., maximal mixing. From this, one can deduce mixing of pairs of configurations as shown in Fig. 13. Also shown in Fig. 13 are mixing strengths from an IBM-MIX calculation [15]. Evidently, the IBM-MIX calculation (which was directed at fitting E2 strengths) seriously fails to describe the pattern of mixing (note that the IBM-MIX calculations are multi-state mixing).

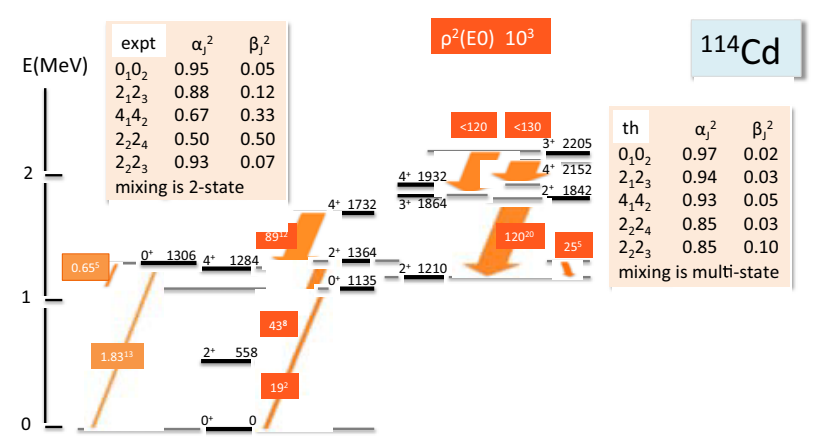

Figure 13. Mixing strengths in ${ }^{114} \mathrm{Cd}$ deduced from electric monopole transition strengths, $\rho^{2}(\mathrm{E} 0)^{*} 10^{3}$ for all strong E0 transitions and comparison with mixing strengths from an IBMMIX calculation [15]. 


\section{Future work and conclusions}

From the emerging pattern of E2 and E0 decay strengths in the mid-shell $\mathrm{Cd}$ isotopes it is evident that considerably more spectroscopic study is needed. A first major direction will be multi-step Coulomb excitation. Such data will identify which excited states are connected by strong E2 transitions. In addition, one- and multi-nucleon transfer data are needed to identify noncollective, i.e., broken pair states.

An issue that impacts nuclear structure more widely and involves the $\mathrm{Cd}$ isotopes is the question; "Do we understand excited $0^{+}$states in nuclei?" An initial exploration of this was made in [16]. It would appear that, from a shell model perspective, the location of shell and subshell gaps is important for answering this question. However, from a more global perspective, the symplectic shell model may provide a unified way forward (see, e.g., [17]).

The author wishes to acknowledge collaborations with Mitch Allmond (Oak Ridge National Lab), Paul Garrett (U. Guelph), Kris Heyde (U. Gent), and Steve Yates (U. of Kentucky) on the study of the Cd istopes.

\section{References}

1. P.E. Garrett, J.L. Wood, J. Phys. G 37, 064028 (2010); err. 069701

2. K. Heyde, J.L. Wood, Rev. Mod. Phys. 83, 1467 (2011)

3. A. Ekström et al., Phys. Rev. C 80, 054302 (2009)

4. S. Ilieva et al., Phys. Rev. C 89, 014313 (2014)

5. P. E. Garrett et al., Phys. Rev. C 64, 024316 (2001)

6. P.E. Garrett et al., Phys. Rev. C 75, 054310 (2007)

7. D. Bandyopadhyay et al., Phys. Rev. C 76, 054308 (2007)

8. M. Kadi et al., Phys. Rev. C 64, 061306 (2003)

9. K.L. Green et al., Phys.Rev. C 80, 032502(R) (2009)

10. P.E. Garrett et al., Phys. Rev. C 86, 044304 (2012)

11. C. Fahlander et al., Nucl. Phys. A 485, 327 (1988)

12. J.L. Wood, E.F. Zganjar, C. De Coster, K. Heyde, Nucl. Phys. A 651, 323 (1999)

13. H.T. Fortune, Phys. Rev. C 35, 2318 (1987)

14. T. Kibedi and R.H. Spear, At. Data Nucl. Data Tables 80, 35 (2002)

15. P.E. Garrett, K.L. Green, J.L. Wood, Phys. Rev. C 78, 044307 (2008)

16. J.L. Wood, J. Phys. Conf. Ser. 403, 012001 (2012)

17. D.J. Rowe, G. Thiamova, J.L. Wood, Phys. Rev. Lett. 97, 202501 (2006) 